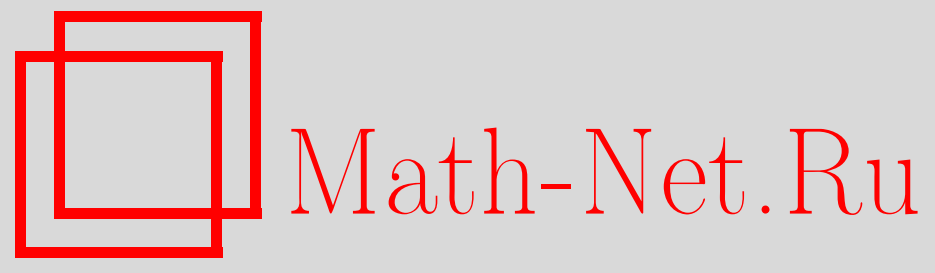

В. П. Радченко, В. В. Цветков, Напряжённодеформированное состояние цилиндрического образца из сплава Д16Т в условиях осевого растяжения и кручения при ползучести, Вестн. Сам. гос. техн. ун-та. Сер. Физ.-мат. науки, 2013, выпуск 3()$, 77-86$

DOI: https://doi.org/10.14498/vsgtu1277

Использование Общероссийского математического портала MathNet.Ru подразумевает, что вы прочитали и согласны с пользовательским соглашением

http://www . mathnet.ru/rus/agreement

Параметры загрузки:

IP : 54.210 .77 .194

26 апреля 2023 г., 14:19:46

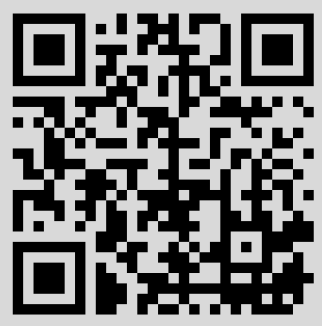


УДК 539.376; 539.434

\title{
НАПРЯЖЁННО-ДЕФОРМИРОВАННОЕ СОСТОЯНИЕ ЦИЛИНДРИЧЕСКОГО ОБРАЗЦА ИЗ СПЛАВА Д16Т В УСЛОВИЯХ ОСЕВОГО РАСТЯЖЕНИЯ И КРУЧЕНИЯ ПРИ ПОЛЗУЧЕСТИ
}

\author{
В. П. Радченко, В. В. Цветков
}

Самарский государственный технический университет, Россия, 443100, Самара, ул. Молодогвардейская, 244.

E-mails: radch@samgtu.ru, v.tsvetkoff@mail.ru

\begin{abstract}
Предложена методика расчёта реологического дебормирования и разрушения иилиндрических образцов в условиях ползучести для трёх видов напряжённого состояния: растяжение, чистое кручение, совместное действие растягивающей нагрузки и крутящего момента. Методика базируется на теории ползучести и длительной прочности энергетического типа. Выполнены расчёты для всех трёх видов напряжённого состояния для сплошных и полых иилиндрических образцов из алюминиевого сплава Д16Т при температуре $250{ }^{\circ} \mathrm{C}$. Проведено сравнение расчётных данных с соответствуюшими эксперименталъными данными при каждом виде напряжённого состояния. Наблюдается соответствие расчётных и экспериментальных значений. Показано, что приложение крутящего момента к растягиваемому осевой нагрузкой образиу интенсифицирует процесс ползучести и приводит к снижению показателей длительной прочности. Наблюдается существенное перераспределение осевых и касательных напряжений по радиусу в зависимости от времени. Приведены оценки погрешностей отклонения расчётных данных от экспериментальных значений.
\end{abstract}

Ключевые слова: ползучесть, длительная прочность, иилиндрический образеи, растяжение, кручение, растяжение с кручением, метод расчёта, экспериментальные данные, сплав Д16Т.

Ужесточение температурно-силовых режимов нагружения, снижение материалоёмкости и использование всех предельных возможностей материала сопротивляться неупругому деформированию и разрушению - центральная задача машиностроения, авиастроения и других отраслей промышленности. Это требует развития методов расчёта элементов конструкций в условиях ползучести с учётом разупрочнения для различных видов напряжённого состояния. Целью данной работы является исследование напряжённо-деформированного состояния сплошного цилиндрического образца в условиях ползучести при совместном действии кручения и растяжения на основе энергетического варианта реологического деформирования и разрушения [1], а также сравнительный анализ данных расчёта по двум различным моделям, представленным в работах $[1,2]$.

Для экспериментальной проверки всех последующих исследований использовались экспериментальные данные по ползучести и длительной прочности для сплава Д16Т при $T=250^{\circ} \mathrm{C}$, приведённые в работе [2] для случаев

Владимир Павлович Радченко (д.ф.-м.н., проф.), заведующий кафедрой, каф. прикладной математики и информатики. Виталий Владимирович Цветков, студент, каф. прикладной математики и информатики. 
растяжения, кручения и совместного растяжения и кручения (первая стадия ползучести у данного материала отсутствует).

\section{1. Одноосная реологическая модель ползучести и длительной прочности.}

В качестве основной модели используется энергетический вариант теории ползучести и длительной прочности [1]. Уравнения состояния этой модели без учёта первой стадии ползучести имеют вид

$$
\dot{p}=c \sigma^{m}, \quad \sigma=\sigma_{0}(1+\omega), \quad \dot{\omega}=\alpha \sigma \dot{p} .
$$

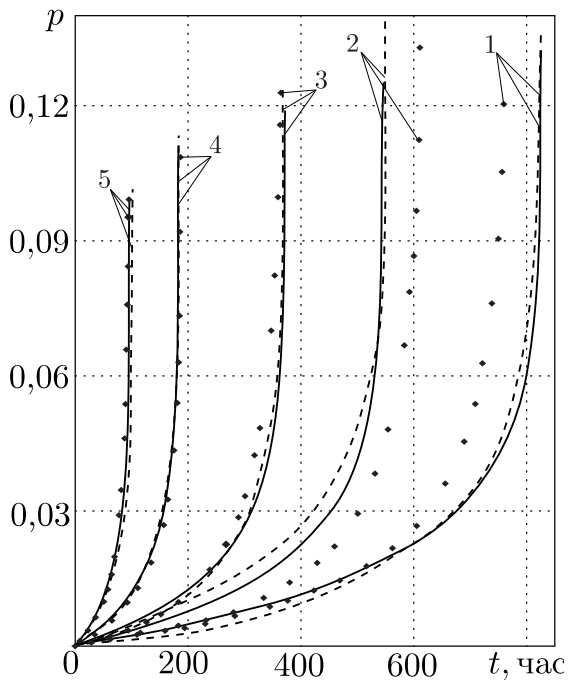

Рис. 1. Экспериментальные (точки) и расчётные кривые ползучести при растяжении для сплава Д16Т при температуре $250^{\circ} \mathrm{C}$ : $1-\sigma_{0}=68,7 \mathrm{M \Pi а;} 2-\sigma_{0}=73,6 \mathrm{M \Pi а} ; 3-$ $\sigma_{0}=78,48 \mathrm{MПа;} 4-\sigma_{0}=88,3 \mathrm{MПа} ; 5-\sigma_{0}=$ $=98,1 \mathrm{M \Pi а}$

Здесь $p$ - деформация ползучести; $\omega-$ параметр повреждённости; $\sigma$ и $\sigma_{0}-$ истинное и номинальное напряжения соответственно; $c, m, \alpha$ - подлежащие определению параметры модели.

В качестве критерия разрушения принимается величина

$$
\Omega(t)=\int_{0}^{t} \frac{\sigma d p}{A_{\mathrm{Kp}}}
$$

где $A_{\text {кр }}$ - критическая величина работы истинных напряжений на деформации ползучести, по достижении которой в момент времени $t=t^{*}$ происходит разрушение материала и $\Omega\left(t^{*}\right)=1$.

В общем случае параметры $\alpha$ и $A_{\text {кр }}$ имеют степенные аппроксимации $\alpha=$ $=\alpha_{1} \sigma_{0}^{m_{\alpha}}, A_{\mathrm{kp}}=A_{1} \sigma_{0}^{m_{A}}$, где $\alpha_{1}, m_{\alpha}$, $A_{1}$ и $m_{A}$ - параметры модели.

В модели (1) и (2) в отличие от модели работы [2] во всех соотношениях используется не номинальное напряжение $\sigma_{0}$, а истинное $\sigma$.

Идентификация параметров $c, m, \alpha_{1}, m_{\alpha}, A_{1}, m_{A}$ хорошо известна [1] и осуществлялась по следующей схеме.

1. С использованием прямолинейных участков кривых ползучести (на начальной стадии $\omega \approx 0$ и $\sigma=\sigma_{0}$ ) методом наименьших квадратов при пяти $\sigma_{0}=$ const определялись параметры степенной аппроксимации $c$ и $m$ первого соотношения (1).

2. Интегрирование первого соотношения (1) при $\sigma_{0}=$ const и начальных условиях $p(0)=0, \omega(0)=0$ даёт зависимость деформации ползучести от $\sigma_{0}$ :

$$
p(t)=-\frac{1}{m \alpha \sigma_{0}} \ln \left|1-\alpha m c \sigma_{0}^{m+1} t\right|
$$

и величину работы истинного напряжения на деформации ползучести:

$$
A(t)=\int_{0}^{t} \sigma d p=\frac{1}{\alpha}\left[\left(1-\alpha m c \sigma_{0}^{m+1} t\right)^{-1 / m}-1\right] .
$$


Параметр $\alpha$ для каждой кривой ползучести, представленной на рис. 1, определялся численно из условия прохождения функции (3) через последнюю точку $\left(t^{*}, p^{*}\right)$. При этом уравнение (3) разрешалось относительно $\alpha$ при заданных $c, m, \sigma_{0}, t^{*}, p^{*}=p\left(t^{*}\right)$ и выполнялась аппроксимация параметра $\alpha$ степенной зависимостью $\alpha=\alpha_{1} \sigma_{0}^{m_{\alpha}}$.

3. Далее для каждой кривой при $\sigma_{0}=$ const по формуле (4) рассчитывалась критическая величина работы разрушения $A_{\mathrm{\kappa p}}=A\left(t^{*}\right)$ и известных $\alpha, m, c$ и строилась степенная зависимость $A_{\mathrm{\kappa p}}=A_{1} \sigma_{0}^{m_{A}}$.

В результате для модели (1), (2) получены следующие параметры: $c=$ $=2,4 \cdot 10^{-15}(\mathrm{MПа})^{-m}, m=5,405, \alpha_{1}=0,91(\mathrm{MПа})^{-m_{\alpha}-1}, m_{\alpha}=-0,43$, $A_{1}=19,72(\mathrm{M \Pi а})^{-1}, m_{A}=0$.

На рис. 1 сплошными линиями показаны результаты расчёта по модели (1), (2), штриховыми линиями - расчёт по методике работы [2], точкамиэкспериментальные данные [2] по ползучести сплава Д16Т при чистом растяжении при $T=250^{\circ} \mathrm{C}$. Анализ информации, представленной на рис. 1 , позволяет сделать вывод о сопоставимости экспериментальных данных и результатов расчёта по моделям, представленным в работах $[1,2]$.

2. Методика расчёта при чистом кручении. Согласно [1], уравнения состояния (1), (2) при введении скалярного параметра повреждённости на случай сложного напряженного состояния для изотропной ползучести обобщаются следующим образом:

$$
\dot{p}_{i j}=\frac{3}{2} c S^{m-1}\left(\sigma_{i j}-\frac{1}{3} \sigma_{k k} \delta_{i j}\right), \quad \sigma_{i j}=\sigma_{i j}^{0}(1+\omega), \quad \dot{\omega}=\alpha\left(S_{0}\right) \sigma_{i j} \dot{p}_{i j} .
$$

Здесь $p_{i j}$ - тензор деформации ползучести; $\sigma_{i j}, \sigma_{i j}^{0}$ - соответственно компоненты истинного и номинального тензоров напряжений; $S, S_{0}$ - соответственно интенсивности тензоров истинных и номинальных напряжений; $c, m-$ константы модели; $\alpha\left(S_{0}\right)$ задаётся степенной аппроксимацией $\alpha\left(S_{0}\right)=\alpha_{1} S_{0}^{m_{\alpha}}$.

Критерий разрушения энергетического типа для сложного напряжённого состояния имеет вид

$$
\Omega\left(t^{*}\right)=\int_{0}^{t^{*}} \frac{\sigma_{i j} d r_{i j}}{A_{\mathrm{\kappa p}}}=1,
$$

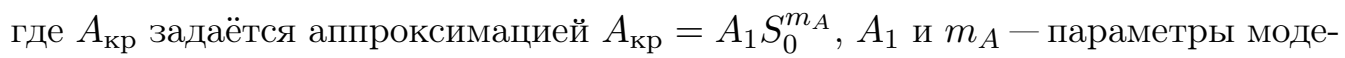
ли.

Все параметры модели (5), (6) для случая изотропной ползучести могут быть определены по результатам одноосных испытаний на растяжение (их значения приведены выше).

Конкретизируем соотношения (5), (6) для частного случая - чистого кручения. Для данного вида напряжённого состояния в работе [2] приведены результаты испытаний, выполненных на тонкостенных образцах из рассматриваемого сплава Д16Т. Соответствующие экспериментальные данные представлены на рис. 2 точками. В [2] отмечается, что сплав Д16Т обладает анизотропией свойств ползучести при растяжении и кручении. Поэтому все параметры модели (5), (6) в условиях кручения будут отличаться от параметров, полученных при растяжении. 


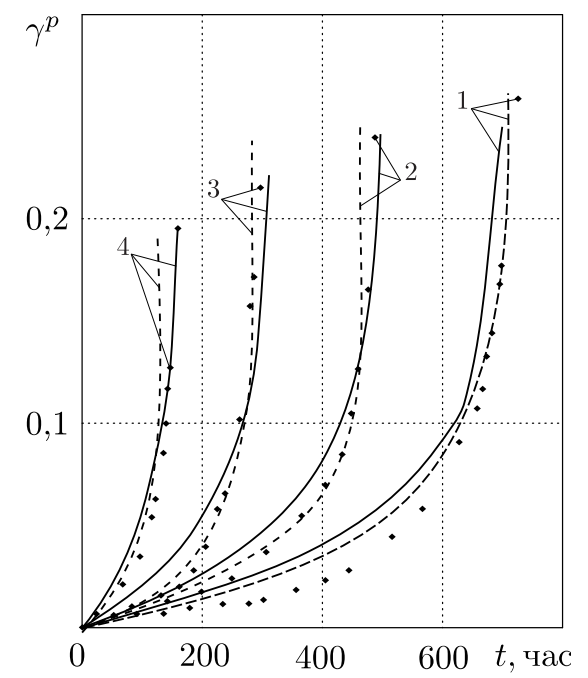

Рис. 2. Экспериментальные и расчётные кривые ползучести для сплава Д16Т на кручение при температуре $250^{\circ} \mathrm{C}: 1-\tau_{0}=$ $=33,94$ МПа; $2-\tau_{0}=36,3$ МПа; $3-\tau_{0}=$ $=39,7$ МПа; $4-\tau_{0}=45,3$ МПа;

Частный случай модели (5), (6) при чистом кручении имеет вид

$$
\begin{aligned}
& \dot{\gamma}^{p}=\sqrt{3} c^{\star}(\sqrt{3} \tau)^{m^{\star}}, \\
& \tau=\tau_{0}(1+\omega), \\
& \dot{\omega}=\alpha^{\star} \tau \dot{\gamma}^{p},
\end{aligned}
$$

где $\gamma^{p}$ - угол сдвига в ползучести; $\tau$, $\tau_{0}$ - истинное и номинальное касательные напряжения; $c^{\star}, m^{\star}, \alpha^{\star}$ - параметры.

Выражение для критерия разрушения при кручении имеет вид

$$
\int_{0}^{t^{\star}} \frac{\tau d \gamma^{p}}{A_{\mathrm{Kp}}^{\star}}=1
$$

где $t^{\star}$ - время разрушения при кручении, $A_{\mathrm{kp}}^{\star}$ - критическая величина работы при кручении.

В силу анизотропии свойств материала Д16Т при растяжении и кручении параметры модели для кручения $c^{\star}$, $m^{\star}, \alpha^{\star}=\alpha_{1}^{\star} S_{0}^{m_{\alpha}^{\star}}, A_{\mathrm{\kappa p}}^{\star}=A_{1}^{\star} S_{0}^{m_{A}^{\star}}\left(S_{0}=\sqrt{3} \tau_{0}\right)$ определяются по результатам испытаний в координатах « $\gamma^{p}-t »$ при $\tau=$ const.

Решение $(7)$ при $\tau_{0}=$ const и начальных условиях $\gamma^{p}(0)=0, \omega(0)=0$ имеет вид

$$
\gamma^{p}=-\frac{1}{\tau_{0} m^{\star} \alpha^{\star}} \ln \left|1-\alpha^{\star} m^{\star} c^{\star}\left(\sqrt{3} \tau_{0}\right)^{m^{\star}+1} t\right|,
$$

а для работы получаем

$$
A(t)=\int_{0}^{t} \tau d \gamma^{p}=\frac{1}{\alpha^{\star}}\left[\left(1-\alpha^{\star} m^{\star} c^{\star}\left(\sqrt{3} \tau_{0}\right)^{m^{\star}+1} t\right)^{-1 / m^{\star}}-1\right] .
$$

Для дальнейших исследований и конкретизации параметров модели (7) используются экспериментальные данные по ползучести при чистом кручении в координатах « $\gamma^{p}-t »$ при $\tau_{0}=$ const для материала Д16Т при $T=$ $=250^{\circ} \mathrm{C}$, приведённые в [2] и представленные точками на рис. 2.

1. С использованием прямолинейных участков кривых ползучести методом наименьших квадратов при четырёх значениях $\tau_{0}$ определялись параметры $c^{\star}$ и $m^{\star}$ степенной аппроксимации первого соотношения (7) (здесь можно считать, что $\tau=\tau_{0}$ ).

2. Далее при известных значениях $m^{\star}$ и $c^{\star}$ и условии прохождения функции (9) через точку разрушения $\left(t^{\star}, \gamma^{\star}\right)$ для каждого значения $\tau_{0}$ определялся параметр $\alpha^{\star}$ и выполнялась аппроксимация $\alpha^{\star}$ зависимостью $\alpha^{\star}=\alpha_{1}^{\star}\left(\sqrt{3} \tau_{0}\right)^{m_{\alpha}^{\star}}$.

3. После определения параметров $c^{\star}, m^{\star}$ и $\alpha^{\star}$ для каждого $\tau_{0}=$ const определялась работа разрушения $A_{\mathrm{\kappa p}}^{\star}=A\left(t^{\star}\right)$ и строилась степенная зависимость $A_{\mathrm{\kappa p}}^{\star}=A_{1}^{\star}\left(\sqrt{3} \tau_{0}\right)^{m_{A}^{\star}}$. 
В итоге получены следующие параметры: $c^{\star}=2,1 \cdot 10^{-15}(\mathrm{MПа})^{-m^{\star}}, m^{\star}=$ $=5,808, \alpha_{1}^{\star}=118,3(\mathrm{M \Pi a})^{-m_{\alpha}^{\star}-1}, m_{\alpha}^{\star}=-1,726, A_{1}^{\star}=214,6(\mathrm{M \Pi a})^{-m_{A}^{\star}-1}$, $m_{A}^{\star}=-0,668$.

На рис. 2 сплошными линиями показаны результаты расчёта $\gamma^{p}=\gamma^{p}(t)$ по модели (7), (8), точками - экспериментальные данные [2], штриховыми линиями - расчётные данные по модели [2].

3. Методика решения краевой задачи о кручении стержня кругового поперечного сечения при ползучести. Рассмотрим стержень кругового поперечного сечения радиуса $R$, к которому приложен крутящий момент $M=M(t)$.

Уравнение равновесия элемента стержня имеет вид

$$
2 \pi \int_{0}^{R} \tau_{0}(r, t) r^{2} d r=M(t)
$$

где $r \in[0, R]$ - расстояние от элемента площади сечения до нейтральной оси, $\tau_{0}(r, t)$ - номинальное напряжение.

Уравнение совместности деформаций в соответствии с гипотезой прямых радиусов принимает вид

$$
\gamma(r, t)=r \theta(t), \quad \gamma(r, t)=\gamma^{e}(r, t)+\gamma(r, t)
$$

где $\gamma(r, t)$ - полный угол сдвига, $\gamma^{e}(r, t)=\tau_{0}(r, t) / G$ - угол сдвига в упругости, $G=E /(2(1+\mu))$ - модуль сдвига материала, $\gamma(r, t)$ - угол сдвига в ползучести, $\theta(t)$ - относительный угол закручивания.

При расчёте весь процесс деформирования разбивается на отрезки $\left[t_{j}, t_{j+1}\right]$ длиной $\Delta t_{j}, j=0,1,2, \ldots$, в пределах которых все характеристики напряжённого состояния считаются постоянными. Тогда для $j$-того отрезка времени можно записать

$$
\gamma\left(r, t_{j+1}\right)=\gamma\left(r, t_{j}\right)+\frac{\Delta \tau_{0 j}(r)}{G}+\Delta \gamma_{j}(r)
$$

где

$$
\Delta \gamma_{j}(r)=\gamma\left(r, t_{j+1}\right)-\gamma\left(r, t_{j}\right), \quad \Delta \tau_{0 j}(r)=\tau_{0}\left(r, t_{j+1}\right)-\tau_{0}\left(r, t_{j}\right) .
$$

Из (10) и второго соотношения (12) получаем

$$
2 \pi \int_{0}^{R} \Delta \tau_{0 j}(r) r^{2} d r=M\left(t_{j+1}\right)-M\left(t_{j}\right)
$$

Соотношения (11) дают

$$
\frac{\Delta \tau_{0 j}(r)}{G}+\Delta \gamma_{j}(r)=r \Delta \theta_{j}
$$

где $\Delta \theta_{j}=\theta\left(t_{j+1}\right)-\theta\left(t_{j}\right)$. Умножая последнее равенство на $2 \pi r^{2} d r$, интегрируя полученное в пределах от 0 до $R$ и используя (13), получаем

$$
\Delta \theta_{j}=\frac{2}{G R^{4}}\left(M\left(t_{j+1}\right)-M\left(t_{j}\right)\right)+\frac{4 \pi}{R^{4}} \int_{0}^{R} \Delta \gamma_{j}^{p}(r) r^{2} d r
$$


Легко видеть, что

$$
\Delta \tau_{0 j}(r)=G\left(r \Delta \theta_{j}-\delta \gamma_{j}^{p}(r)\right)
$$

Далее получаем все искомые характеристики по следующей схеме. Приращения неупругих деформаций при фиксированном $r$ на отрезке $\left[t_{j}, t_{j+1}\right]$ определяются по формулам (14), (15). При этом неупругие деформации и параметр повреждённости имеют нулевые начальные условия. Величины $\gamma^{p}, \tau_{0}$, $\omega, \theta$ при $t \in\left[t_{j}, t_{j+1}\right]$ определяются по рекуррентному соотношению

$$
F\left(t_{j+1}\right)=F\left(t_{j}\right)+\Delta F_{j}
$$

где $F$ - любая из этих величин.

Расчёт ползучести стержня осуществляется до тех пор, пока выполняется условие

$$
\Omega(t)=\int_{0}^{t} \frac{\tau d \gamma^{p}}{A_{1}^{\star}\left(\sqrt{3} \tau_{0}\right)^{m_{A}^{\star}}}<1 .
$$

Если при каком-либо значении радиуса стержня выполняется условие $\Omega\left(t^{\star}\right)=$ $=1$, то предполагается, что при $t=t^{\star}$ происходит разрушение образца.

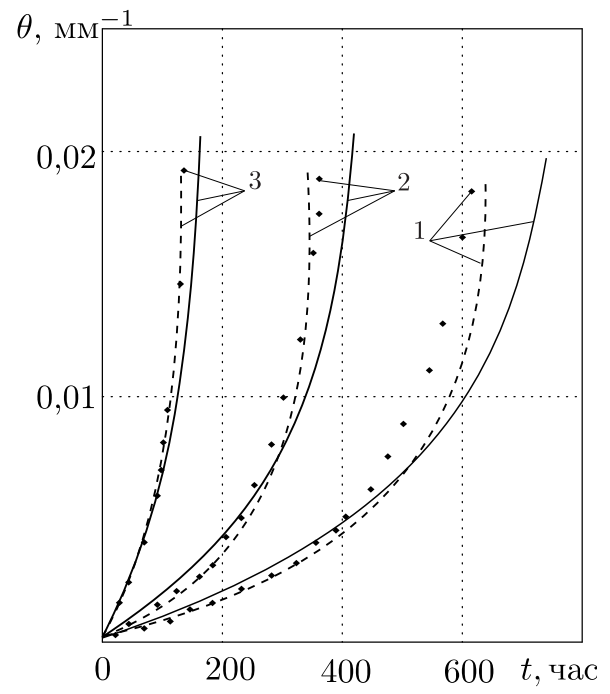

Рис. 3. Экспериментальные и расчётные значения погонного угла закручивания стержня из сплава Д16Т при темпеpaтуре $250{ }^{\circ} \mathrm{C}: 1-M=69,3 \mathrm{\kappa H} \cdot \mathrm{мм} ; 2-$ $M=77,05 \mathrm{\kappa H} \cdot \mathrm{мм} ; 3-M=92,45 \mathrm{\kappa H} \cdot \mathrm{мм}$

В качестве примера был рассмотрен случай неупругого деформирования и разрушения цилиндрического образца кругового поперечного сечения с радиусом $R=10$ мм из сплава Д16Т при температуре $T=250^{\circ} \mathrm{C}$. Экспериментальные данные для указанного цилиндра приведены в [2]. Все параметры реологической модели (7), (8) приведены выше, а значения модуля Юнга и коэффициента Пуассона следующие: $E=56000$ МПа, $\mu=0,3$.

На рис. 3 сплошными линиями представлены расчётные данные по модели (7), (8), (10)-(15), точками экспериментальные значения погонного угла закручивания $\theta=\theta(t)$ для трёх величин крутящего момента $M=\{69,3 ; 77,05 ; 92,45\}$ кН $\cdot$ мм. Здесь же штриховыми линиями показаны расчётные данные по модели из работы [2].

На рис. 4 и 5 представлено распределение номинальных и истинных касательных напряжений соответственно по радиусу стержня в различные моменты времени при $M=92,45 \mathrm{kH} \cdot$ мм.

Как следует из информации, представленной на рис. 3, наблюдается удовлетворительное соответствие расчётных и экспериментальных значений деформации ползучести в координатах «относительный угол закручивания $\theta-$ 


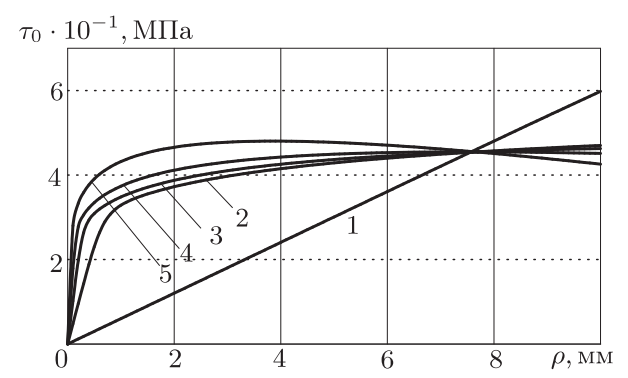

Рис. 4. Распределение номинального касательного напряжения по радиусу стержня в различные моменты времени при $M=92,45 \mathrm{kH} \cdot \mathrm{мм}: 1-t=0$ ч; $2-t=38$ ч; $3-t=76$ ч; $4-t=114$ ч; $5-t=152$ ч

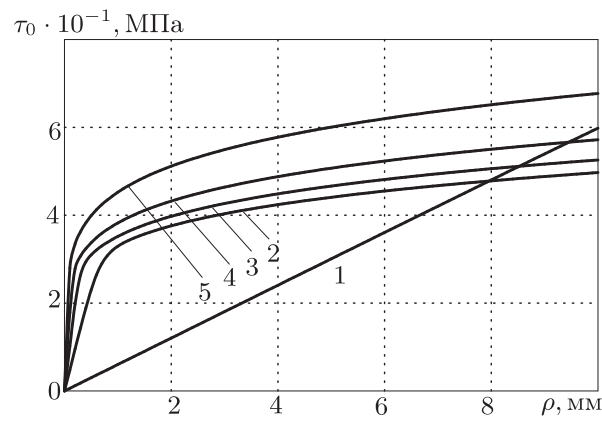

Рис. 5. Распределение истинного касательного напряжения по радиусу стержня в различные моменты времени при $M=92,45 \mathrm{\kappa H} \cdot \mathrm{мм}: 1-t=0$ ч; $2-t=38$ ч; $3-t=76$ ч; $4-t=114$ ч; $5-t=152$ ч

время $t$ » при различных значениях крутящего момента. Из рис. 4 и 5 следует, что при ползучести происходит существенное перераспределение номинального и истинного касательных напряжений по радиусу в зависимости от времени.

4. Решение краевой задачи о реологическом деформировании цилиндрического образца при совместном растяжении и кручении. Рассмотрим реологическую модель ползучести при совместном действии растяжения и кручения с учётом отмеченной анизотропии свойств для этих видов напряжённого состояния. Исходя из общей модели при сложном напряжённом состоянии и одномерных моделей частного вида (1) и (7) для случая анизотропной ползучести определяющие соотношения можно принять в следующем виде:

$$
\begin{array}{lll}
\dot{p}=c S^{m-1} \sigma, & \dot{\gamma}=3 c^{\star} S^{m^{\star}-1} \tau, & \sigma=\sigma_{0}(1+\omega), \\
\tau=\tau_{0}(1+\omega), & S_{0}=\sqrt{\sigma_{0}^{2}+3 \tau_{0}}, & S=S_{0}(1+\omega), \\
\dot{\omega}=\alpha \sigma \dot{p}+\alpha^{\star} \tau \dot{\gamma}^{p}, & \alpha=\alpha_{1}\left(S_{0}\right)^{m_{\alpha}}, & \alpha^{\star}=\alpha_{1}^{\star}\left(S_{0}\right)^{m_{\alpha}^{\star}},
\end{array}
$$

где $p$-осевая деформация ползучести, $\gamma^{p}$-угол сдвига в ползучести, $\sigma$ и $\sigma_{0}$ - истинные и номинальные нормальные напряжения, $\tau$ и $\tau_{0}$ - истинные и номинальные касательные напряжения, $S$ и $S_{0}$ - интенсивности истинных и номинальных напряжений; все остальные параметры определяются моделями (1), (2) и (7), (8).

Соответственно изменится и критерий разрушения. Расчёт осуществляется до тех пор, пока выполняется условие

$$
\Omega(t)=\int_{0}^{t} \frac{\sigma d p}{A_{1}\left(S_{0}\right)^{m_{\alpha}}}+\int_{0}^{t} \frac{\tau d \gamma}{A_{1}^{\star}\left(S_{0}\right)^{m_{\alpha}^{\star}}}<1 .
$$

Очевидно, что модели (1), (2) и (7), (8) являются частными случаями модели (16), (17) при $\tau_{0}=0$ и $\sigma_{0}=0$ соответственно.

Рассмотрим решение краевой задачи о ползучести полого толстостенного цилиндрического образца с внутренним радиусом $R_{1}$ и внешним $R_{2}$ при совместном действии растягивающей нагрузки $Q$ и крутящего момента $M$. 
Запишем уравнения равновесия и совместности деформаций с использованием гипотезы плоских сечений и прямых радиусов:

$$
\begin{aligned}
2 \pi \int_{R_{1}}^{R_{2}} \sigma_{0}(r, t) r d r & =Q(t), \quad \frac{\sigma_{0}(r, t)}{E}+p(r, t)=\varepsilon_{0}(t), \\
2 \pi \int_{R_{1}}^{R_{2}} \tau_{0}(r, t) r^{2} d r & =M(t), \quad \frac{\tau_{0}(r, t)}{G}+\gamma^{p}(r, t)=r \theta(t),
\end{aligned}
$$

где $r \in\left[R_{1}, R_{2}\right]$ - расстояние от элемента площади до оси цилиндра, $\theta(t)$ относительный угол закручивания, $\varepsilon_{0}(t)$ - осевая деформация образца.

Решение задачи (18), (19) на основе реологической модели (16), (17) осуществляется численно «шагами» по времени аналогично случаю чистого изгиба.

В качестве примера рассмотрим результаты расчёта ползучести полого цилиндрического образца из сплава Д16Т $\left(T=250^{\circ} \mathrm{C}\right)$ при $R_{1}=5 \mathrm{мм}, R_{2}=$ $=10$ мм и различных комбинациях $Q$ и $M$, а также сравним их с соответствующими экспериментальными данными, представленными в работе [2]. На рис. 6 и рис. 7 сплошными линиями показаны расчётные значения осевой деформации ползучести $p=p(t)$ при различных значениях растягивающей нагрузки $Q$ и крутящего момента $M$, на рис. 8 приведена кинетика перераспределения номинальных напряжений $\sigma_{0}=\sigma_{0}(r)$ и касательных напряжений $\tau_{0}=\tau_{0}(r)$ в различные моменты времени при $Q=18,48 \mathrm{kH}, M=47,2 \mathrm{kH} \cdot$ мм.

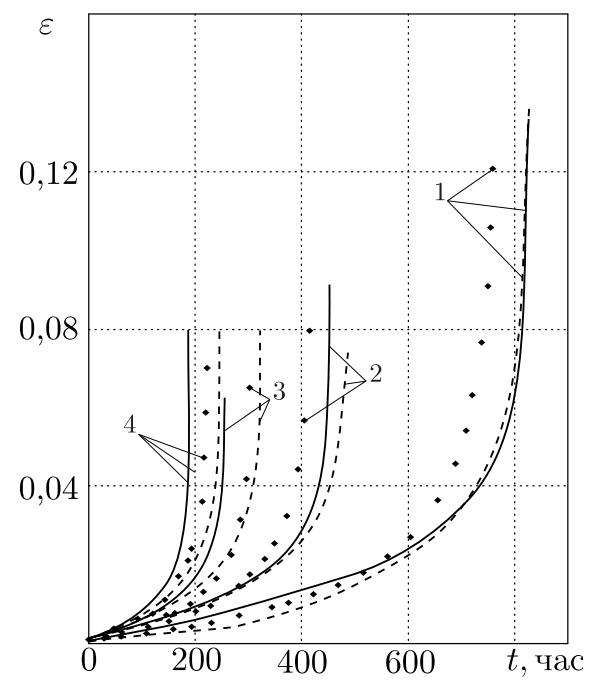

Рис. 6. Осевая деформация ползучести полого цилиндрического образца из сплава Д16Т $\left(T=250{ }^{\circ} \mathrm{C}\right)$ при осевой нагрузке $Q=16,17 \mathrm{\kappa H}$ и различных значениях крутящего момента: $1-M=0,2-M=$ $=28,9 \mathrm{\kappa H} \cdot \mathrm{мм}, 3-M=43,3 \mathrm{\kappa H} \cdot \mathrm{мм}, 4-M=$ $=50,6 \mathrm{kH} \cdot \mathrm{мм} ;$ точки - экспериментальные данные [2]; сплошные линии - расчёт по модели (16)-(19); штриховые линии - расчёт по модели [2]

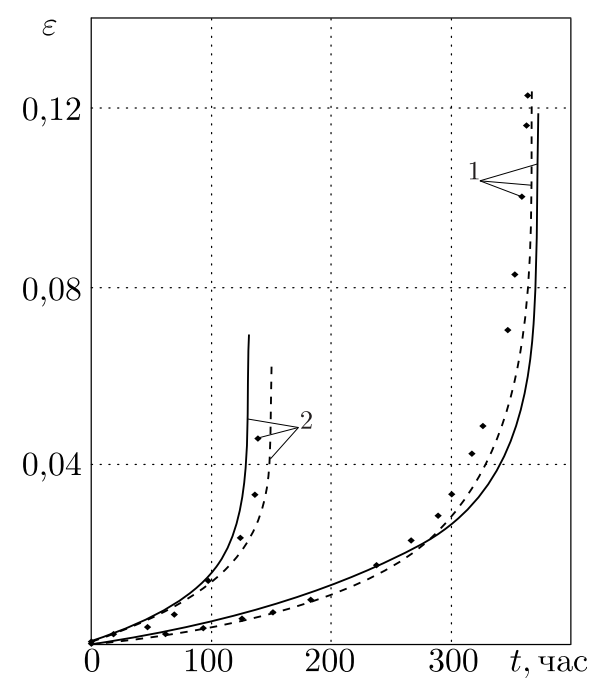

Рис. 7. Осевая деформация ползучести полого цилиндрического образца из сплава Д16Т $\left(T=250{ }^{\circ} \mathrm{C}\right)$ при осевой нагрузке $Q=$ $=18,48 \mathrm{\kappa H}$ и различных значениях крутящего момента: $1-M=0,2-M=47,2 \mathrm{\kappa H}$. . мм; точки - экспериментальные данные [2]; сплошные линии - расчёт по модели (16)-(19); штриховые линии - расчёт по модели [2] 

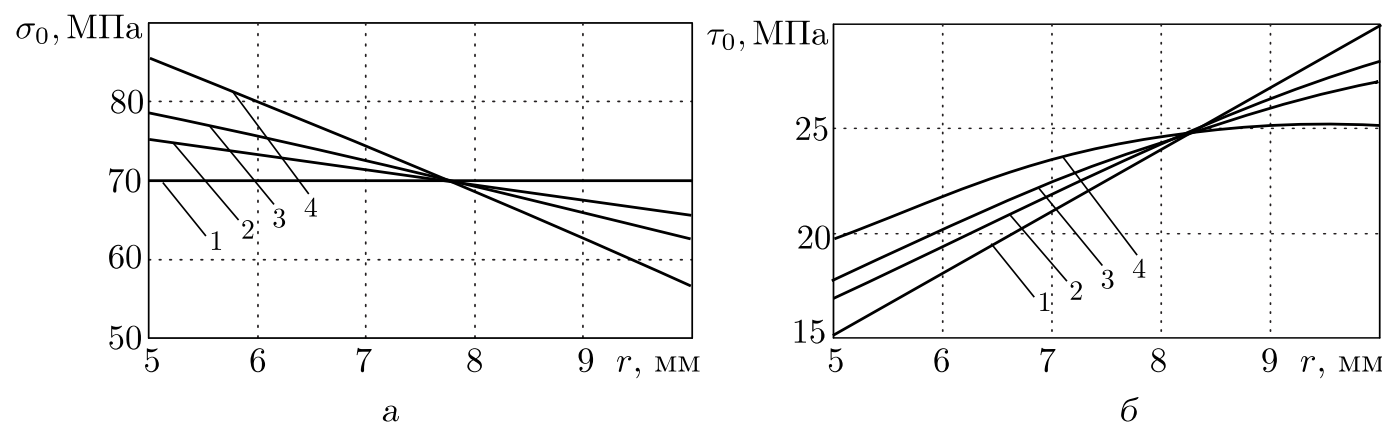

Рис. 8. Распределение номинальных напряжений $\sigma_{0}=\sigma_{0}(r)$ (а) и номинальных касательных напряжений $\tau_{0}=\tau_{0}(r)(б)$ в различные моменты времени при $Q=18,48 \mathrm{kH}$, $M=47,2 \mathrm{KH} \cdot \mathrm{мм}: 1-t=0 ; 2-t=100$ ч; $3-t=200$ ч; $4-t=250$ ч

\begin{tabular}{c|c|c|c|c}
\hline$Q, \mathrm{kH}$ & $M, \mathrm{\kappa H} \cdot \mathrm{MM}$ & $t_{\ni}^{*}$, ч & $t_{\mathrm{p}}^{*}$, ч & $t_{\mathrm{p}}^{*}$, ч [2] \\
\hline 16,17 & 0 & 760 & 825 & 827 \\
16,17 & 28,9 & 416 & 453 & 430 \\
16,17 & 43,3 & 303 & 255 & 323 \\
16,17 & 50,6 & 223 & 187 & 247 \\
18,48 & 0 & 365 & 371 & 368 \\
18,48 & 47,2 & 143 & 132 & 151 \\
\hline
\end{tabular}

Анализ этих данных позволяет сделать вывод, что приложение крутящего момента к нагруженному осевой нагрузкой цилиндрическому образцу существенно интенсифицирует процесс ползучести и приводит к перераспределению во времени не только касательных напряжений по радиусу, но и нормальных напряжений. С целью сопоставления результатов расчётов в таблице для каждой комбинации растягивающей осевой силы $Q$ и крутящего момента $M$ приведены экспериментальные $t_{\ni}^{*}$ и расчётные значения $t_{\mathrm{p}}^{*}$ времени разрушения по модели (16)-(19) и по модели работы [2]. Ошибка отклонения расчётного времени разрушения от экспериментального вычислялась по формуле

$$
\Delta=\frac{1}{N} \sum_{i=1}^{N}\left|\frac{t_{\ni i}^{*}-t_{\mathrm{p} i}^{*}}{t_{\ni i}^{*}}\right| \cdot 100 \%,
$$

где $N=6$ - число вариантов расчёта, и для модели (16)-(19) составила величину $9,8 \%$, а для модели, предложенной в [2],-8,4\%. В целом наблюдается удовлетворительное соответствие расчётных данных по предложенной авторами методике экспериментальным данным и данным расчёта по модели работы [2].

\section{БИБЛИОГРАФИЧЕСКИЙ СПИСОК}

1. В. П. Радченко, Ю. А. Ерёмин, Реологическое деформирование и разрушение элементов конструкций. М.: Машиностроение-1, 2004. 264 с. [V. P. Radchenko, Yu. A. Eremin, Rheological Deformation and Fracture of Materials and Structural Elements. Moscow: Mashinostroenie-1, 2004. 264 pp.]

2. О. В. Соснин, Б. В. Горев, А. Ф. Никитенко, Энергетический вариант теории ползучести. Новосибирск: Институт гидродинамики СО РАН СССР, 1986. 95 с. [O. V. Sosnin, 
B. V. Gorev, A. F. Nikitenko, Energy variant of creep theory. Novosibirsk: Inst. of Hydrodynamics, USSR Acad. of Sci., 1986. 95 pp.]

Поступила в редакцию 24/VI/2013;

в окончательном варианте - 24/VII/2013.

MSC: 74D10

\title{
THE STRESS-STRAIN STATE OF CYLINDRICAL SAMPLE FROM ALLOY D16T UNDER AXIAL TENSION AND TORSION CREEP
}

\author{
V. P. Radchenko, V. V. Tsvetkov
}

Samara State Technical University,

244, Molodogvardeyskaya st., Samara, 443100, Russia.

E-mails: radch@samgtu.ru, v.tsvetkoff@mail.ru

The method for calculation of cylindrical sample rheological deformation and fracture in creep conditions for three types of stress state: tension, pure torsion, the combined effect of tensile load and torque is offered. The procedure is based on the theory of creep and creep rupture strength of energy type. Calculations are performed for all three types of stress state for solid and hollow cylinder tests from aluminum alloy D16T at $250^{\circ} \mathrm{C}$. Comparison of the calculated data with the corresponding test data for each type of stress state is conducted. It shows the agreement between the calculated and experimental values. The intensification of creep and decreasing of creep rupture strength factors after the application of torque to the specimen under the axial tension are established. The substantial redistribution of axial and shear stresses along the radius depending on the time is observed. The estimates of errors of deviation of calculated data from the experimental values are given.

Key words: creep, creep rupture strength, cylindrical sample, tension, torsion, tension with torsion, the method of calculation, test data, alloy D16T.

Original article submitted 24/VI/2013; revision submitted $24 / \mathrm{VII} / 2013$.

Vladimir P. Radchenko (Dr. Phys. \& Math. Sci.), Head of Dept., Dept. of Applied Mathematics \& Computer Science. Vitaliy V. Tsvetkov, Student, Dept. of Applied Mathematics \& Computer Science. 